\title{
Zur Bodenentwicklung auf Moränen in den Alpen
}

\section{Problemstellung und Arbeitsgebiet}

Trotz immer genauerer Kenntnisse über das Verhalten der Gletscher im Postglazial aufgrund von Moränenkartierungen, Pollenanalysen, Dendrochronologie, Vergleich mit alten Stichen und Karten, Gletschervermessung und 14C-Daten sind wir noch immer von einer detaillierten Rekonstruktion des Geschehens der letzten 10000 Jahre entfernt. Es sei speziell auf die vielen 14C-Daten verwiesen, die in oft verführerischer Weise absolute Altersangaben vortäuschen, auch wenn es sich für die eingestuften Prozesse und Formen immer nur um ein Maximalbzw. Minimalalter handeln kann. Bei den vorliegenden Untersuchungen bestand die Absicht, in einem morphologisch bereits kartierten Gebiet eine Relativdatierung von Moränen mit Hilfe der auf ihnen entwickelten Böden vorzunehmen. Zwei Grundvoraussetzungen müssen dafür erfüllt sein: Erstens dürfen die Moränen nicht selbst schon aus vorverwittertem Material bestehen, und zweitens muß die Pedogenese unmittelbar an die Morphogenese anschließen und ununterbrochen bis heute andauern. Gerade dieser zweite Punkt scheint häufig im alpinen Raum nicht oder nur bedingt erfüllt zu sein.

Was die Literatur zum Zeitfaktor in der Bodenentwicklung betrifft, so sind die Meinungen dazu etwas widersprüchlich, aber nicht grundsätzlich voneinander verschieden. Im alpinen Raum kann offensichtlich damit gerechnet werden, daß auf frühneuzeitlichen Moränen bereits visuell eine deutliche Bodenbildung erfolgt ist, beispielsweise eine schwache Podsolierung am Steingletscher (KING, 1974) oder eine junge Rasenbraunerde im Großglocknergebiet (BURGER, 1972). Auch ZECH und WILKE (1977) kommen im Zillertal zum Schluß, daß sich alpine Podsole nach etwa 600 Jahren bilden können. Andrerseits aber weisen MAYR (1964) und PATZELT (1973) darauf hin, daß auf den frühneuzeitlichen Moränen höchstens Rankerbildungen anzutreffen sind! Im Ostseeraum weisen die Autoren AALTONEN für Finnland (in JAUHIAINEN, 1972b), JAUHIAINEN für Polen (1969) sowie BERGQVIST und LINDSTROEM für Schweden (in JAUHIAINEN, 1972b) nach, daß für eine sichtbare Podsolierung rund 500 Jahre erforderlich sind. Dem Problem der Weiterentwicklung eines einmal entstandenen Bodens wird jedoch, was für unsere Untersuchungen wichtig wäre, kaum Beachtung geschenkt.
Die Wahl eines Gletschervorfeldes im weiteren Sinn als Testgebiet kann nach Abschluß der Untersuchungen nicht mehr voll unterstützt werden, ergibt doch die Gesamtheit aller Moränen des Spät- und Postglazials kaum eine zeitliche gleichmäßige Abfolge, sondern eher zwei bis drei Altersgruppen: Egesen(und evtl. Daun-)stände im Spätglazial sowie die Stände der letzten rund 1000 Jahre (oder möglicherweise des ganzen Postglazials) in der neuzeitlichen Größenordnung.

Das Morteratschgebiet (Abb. 1) wurde gewählt, weil hier (außer der bereits bestehenden geomorphologischen Aufnahme von BEELER, 1977) die ältesten und jüngsten Moränen auf engstem Raum beieinander liegen, womit die bodenbildenden Faktoren Klima, Vegetation (subalpiner Nadelwald mit Zwergsträuchern) und Ausgangsmaterial (Kristallin des Kerns der Berninadecke) als durchaus vergleichbar angesprochen werden können. Im ganzen Untersuchungsgebiet (mit Ausnahme des eigentlichen Gletschervorfeldes und der Flußniederung) findet sich der Bodentyp des Eisenpodsols.

In diesem Testgebiet wurden 16 Bodenprofile (Lokalitäten A bis P in Fig. 1) in die detaillierte Untersuchung einbezogen. $\mathrm{Da}$ damit natürlich keine repräsentative Aussage gemacht werden kann, ist klar, doch sollten so gewisse Trends bei den Untersuchungen bereits zum Ausdruck kommen (wobei immer mit einzelnen "Ausreißerprofilen» gerechnet werden muß). Ein Spezialfall ist die Lokalität "M» (Fig. 2, Abb. 2). Hier liegen sicher 4 Moränen nahe bei- oder sogar übereinander. Leider kann die Situation nur noch rekonstruiert werden, da ein Großteil des Moränenmaterials für Bachverbauungen benötigt worden ist. Eindeutig lassen sich die grobblockigen Moränen aus der Mitte des letzten Jahrhunderts identifizieren. Diese Wälle wurden offensichtlich an eine ältere Moräne «Mb» angelagert, die ihrerseits wiederum einer noch älteren Moräne «Ma» aufsitzt. Während diese Moräne, deren Alter unbekannt ist, einen optimal entwickelten Podsol besitzt, befindet sich auf «Mb» ein nur schwach ausgebildeter Podsol, und auf den Moränen von 1850 haben sich nur Ranker, andeutungsweise an einigen Stellen etwas verbraunt, entwickelt.

Dr. Peter Fitze, Geographisches Institut der Universität Zürich Postfach, 8033 Zürich. 


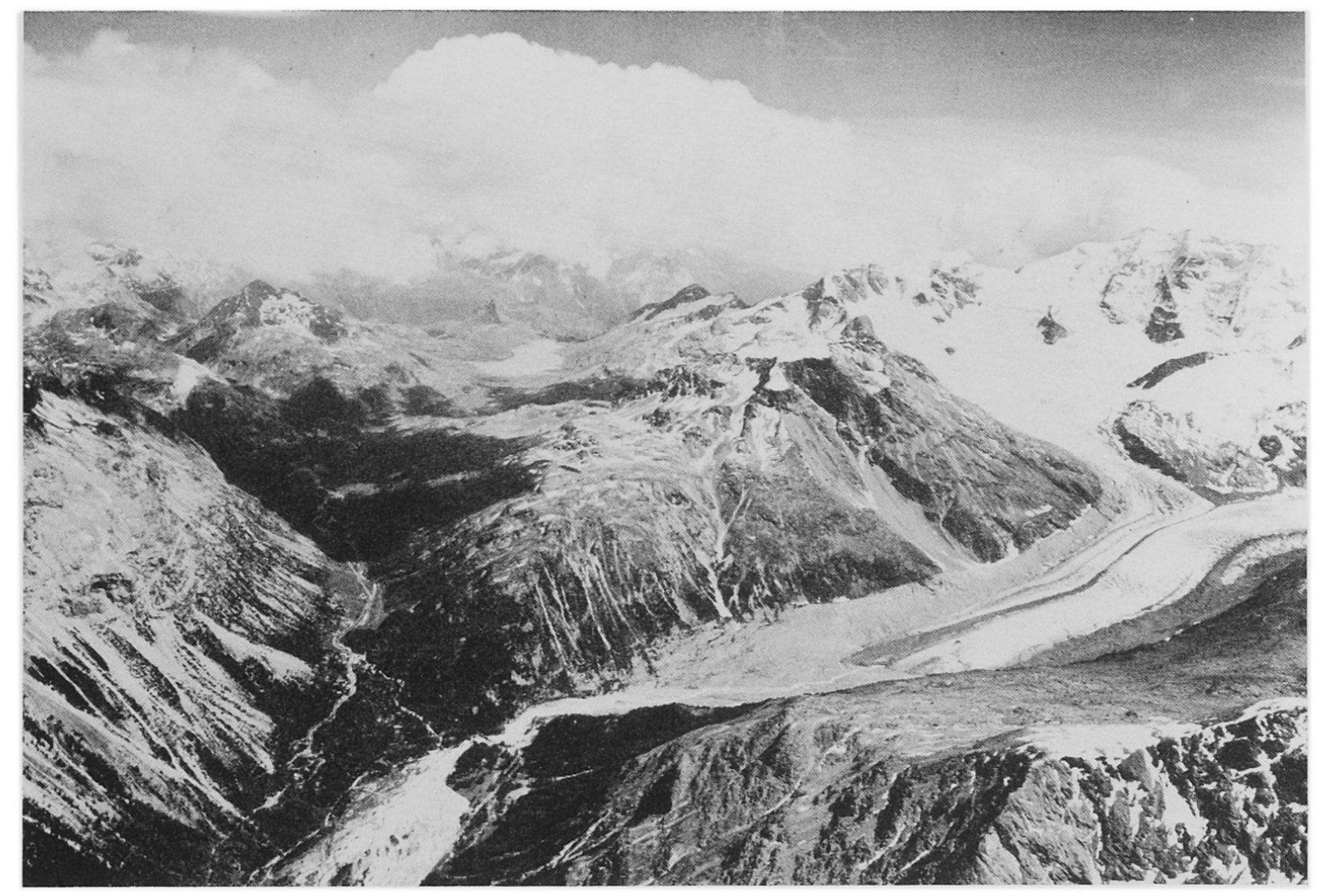

Abb. 1 Blick von Nordwesten auf die Regionen des Berninapasses. Rechts im Bild der Piz Palü, davor der vereinigte Pers-Morteratschgletscher mit der deutlich erkennbaren Seitenmoräne aus dem 19. Jahrhundert. Die bewaldete Mündung des Berninatales (im Vordergrund links) bildet das Untersuchungsgebiet im engeren Sinn. Aufnahme: Swissair Photo, rep. mit Bewilligung vom 30.6.1980.

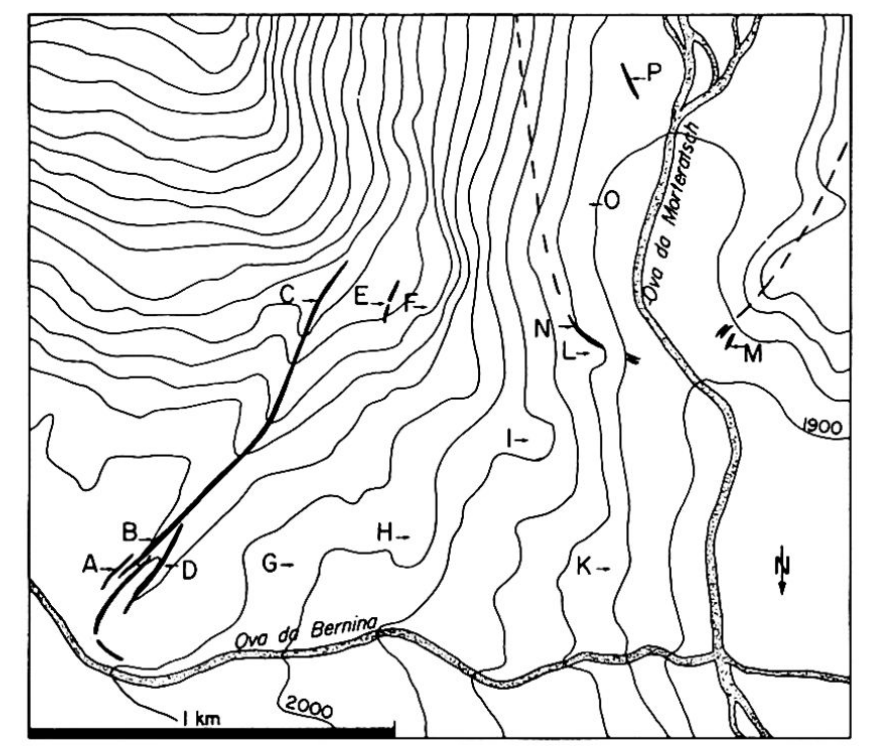

Fig. 1 Skizze des Morteratschgebietes mit den untersuchten Lokalitäten. Klar zu erkennen sind die spätglazialen Stände A bis $D$, die ins Berninatal reichen, sowie das rezente Gletschervorfeld mit den Rankerprofilen N bis P. Die alphabetische Reihenfolge gibt die wahrscheinlichste Chronosequenz an, wobei verschiedentlich Gleichaltrigkeit nicht ausgeschlossen werden kann.
Falls es gelänge, $\mathrm{Mb}$ zeitlich einzustufen, wäre in dieser Gegend zumindest ein Zeitpunkt festgelegt für die Entwicklung bis zu einem schwach ausgeprägten Podsol. Der Podsol von Ma zieht fast ungestört unter die Moräne $\mathrm{Mb}$, wo er fossilisiert wurde. Aus dem Ah-Horizont (Abb. 2) wurde ein 14C-Datum von $1143 \pm 44$ Jahren BP ermittelt (UZ-256). Da sich bei der Bestimmung eines humosen Mineralbodens immer ein Mischalter ergibt, kann aus dieser $14 \mathrm{C}$-Bestimmung gefolgert werden, daß die Moräne $\mathrm{Mb}$ mit größter Wahrscheinlichkeit nicht älter als etwa 1000 Jahre alt sein kann. Es ist sogar denkbar, daß dieser Wall in die Frühneuzeit, also ins 17. Jahrhundert, gestellt werden kann. Dann allerdings würde die doch schon recht fortgeschrittene Bodenbildung von $\mathrm{Mb}$ im Vergleich zu den noch unentwickelten Böden auf den Moränen des 19. Jahrhunderts verblüffen (pH-Werte, org. $\mathrm{C}$ und die hier nicht dargestellten pedogenen Oxide), es sei denn, daß es sich beim Moränenmaterial von $\mathrm{Mb}$ um vom Gletscher aufgeschobenes vorverwittertes Material gehandelt hätte. Auf jeden Fall zeigt die Lokalität $\mathrm{M}$ eine überraschende Parallelität zum Vorfeld des Steingletschers (KING, 1974), wo die jüngere Moräne ins 17. Jahrhundert gestellt wurde. 


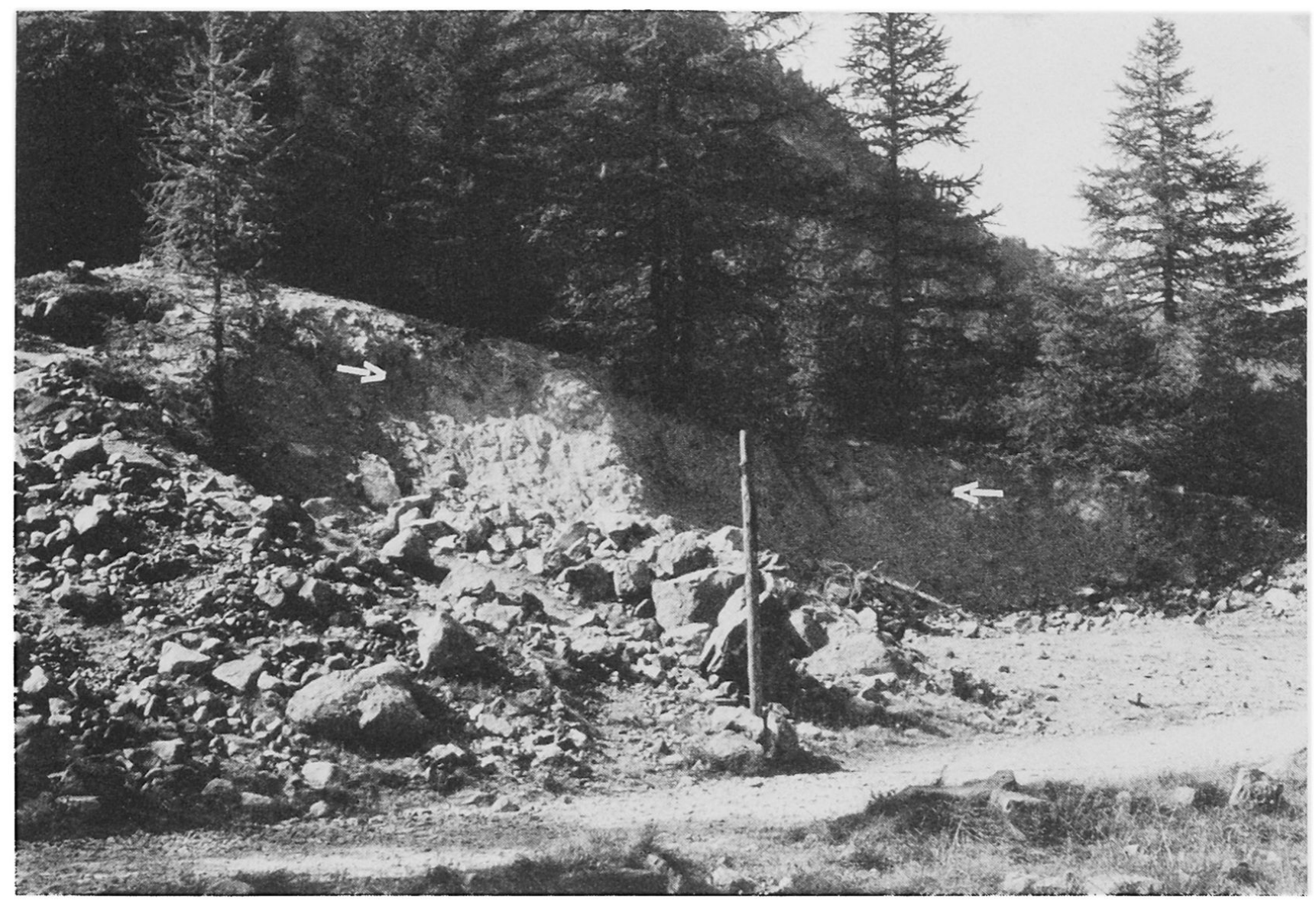

Abb. 2 Die Lokalität «M» von der Innenseite her gesehen. Es sind die zwei übereinander liegenden Moränen Ma und Mb zu erkennen (fossiler Ah-Horizont von Ma durch Pfeile gekennzeichnet). Die Blöcke im Vordergrund gehören zu den Moränen des 19. Jahrhunderts. Aufnahme J. Suter.

Um sich eine Vorstellung über die Bodenbildungen im Morteratschgebiet machen zu können, ist in Fig. 3 ein Sammelprofil eines Eisenpodsols dargestellt, das recht klar die wichtigsten Eigenheiten dieses Bodentyps beleuchtet:

- starke Versauerung im Bereich des Oberbodens und des Auswaschungshorizontes (Ah- und E-Horizont).

- Verlagerung des organischen Materials vom E- in den Bfe-Horizont.

- Verlagerung des bei der Verwitterung freigesetzten Eisens und Aluminiums $\left(\mathrm{Fe}_{d}, \mathrm{Al}_{\mathrm{d}}\right)$ vom $\mathrm{E}$ - in den Bfe-Horizont. Diese Verlagerung dürfte sich vornehmlich mit der org. Substanz in Form von metall-

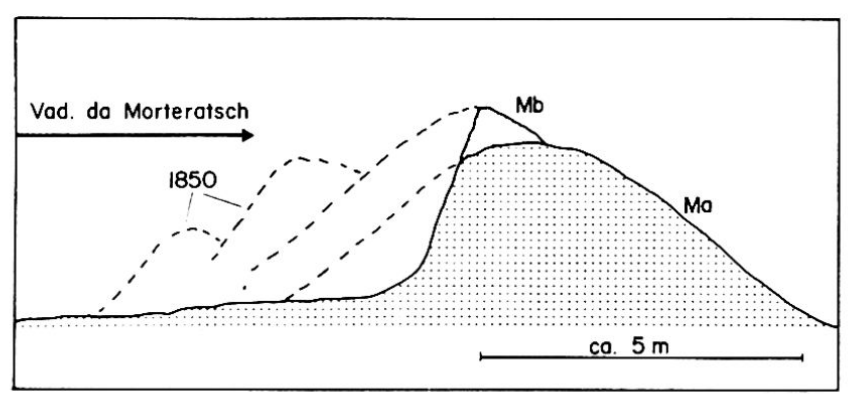

Fig. 2 Schematische Skizze der Lokalität «M» organischen Komplexen abspielen. Ausdruck dieser Verlagerungsprozesse ist die Bleichung des Eund eine intensive Rostfärbung des Bfe-Horizontes.

\section{Analytische Untersuchungen und mögliche Gliederung}

Nachdem sich erwartungsgemä $ß$ gezeigt hatte, da $\beta$ bei den Podsolbildungen im Untersuchungsgebiet visuelle Kriterien wie etwa Farbintensität oder Horizontmächtigkeiten nicht zu einer Untergliederung führten, wurde eine Auswahl von chemischen Analysen getroffen, die den pedogenetischen Prozessen am ehesten Rechnung tragen könnten. Das Spektrum an Möglichkeiten könnte natürlich noch beliebig erweitert werden. Wegen der vorherrschenden Podsolierung im Arbeitsgebiet richtete sich das Hauptinteresse auf die Prozesse der Versauerung sowie der Verlagerung von Humusstoffen und Sesquioxiden. Es muß an dieser Stelle mit Nachdruck darauf hingewiesen werden, daß sich die bestimmten Gehalte an Humus oder auch an Sesquioxiden nur auf die Feinerde beziehen. Es fehlt somit der an und für sich richtigere Volumenbezug, was bei unseren blockreichen Moränen, in denen die Feinderde oft nur den 

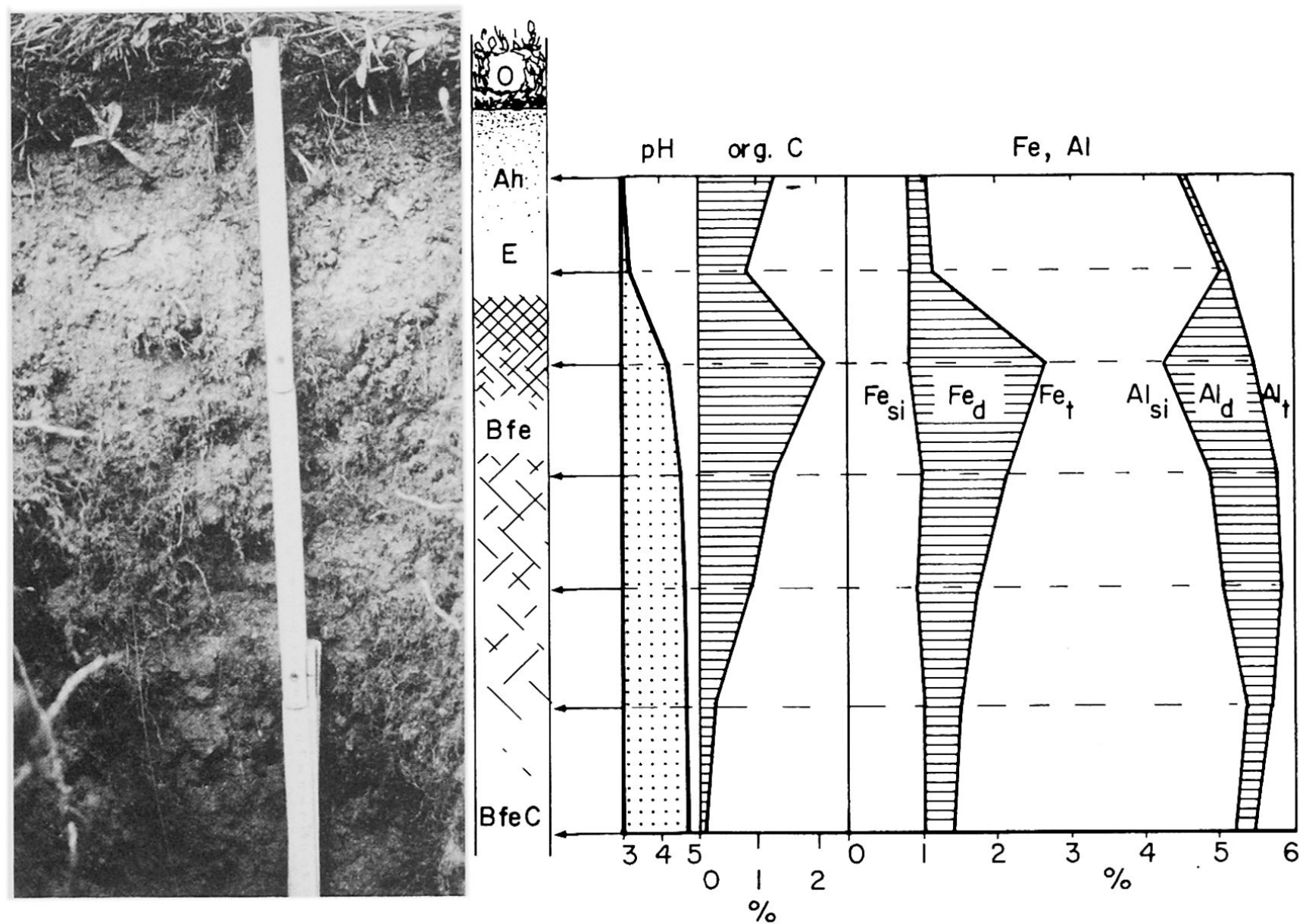

Fig. 3 Typisches Erscheinungsbild eines Podsols aus dem Morteratschgebiet sowie Sammelprofil einiger Analysendaten (Mittelwert von sechs Profilen). Die Analysendaten sind gewichtsbezogen auf die Feinerde. Die Fraktionen $\mathrm{Fe}_{s i}$ und $\mathrm{Al}_{s i}$ wurden als Differenz $\mathrm{Fe}_{t}-\mathrm{Fe}_{\mathrm{d}}$ und $\mathrm{Al}_{\mathrm{t}}-\mathrm{Al}_{\mathrm{d}}$ bestimmt.

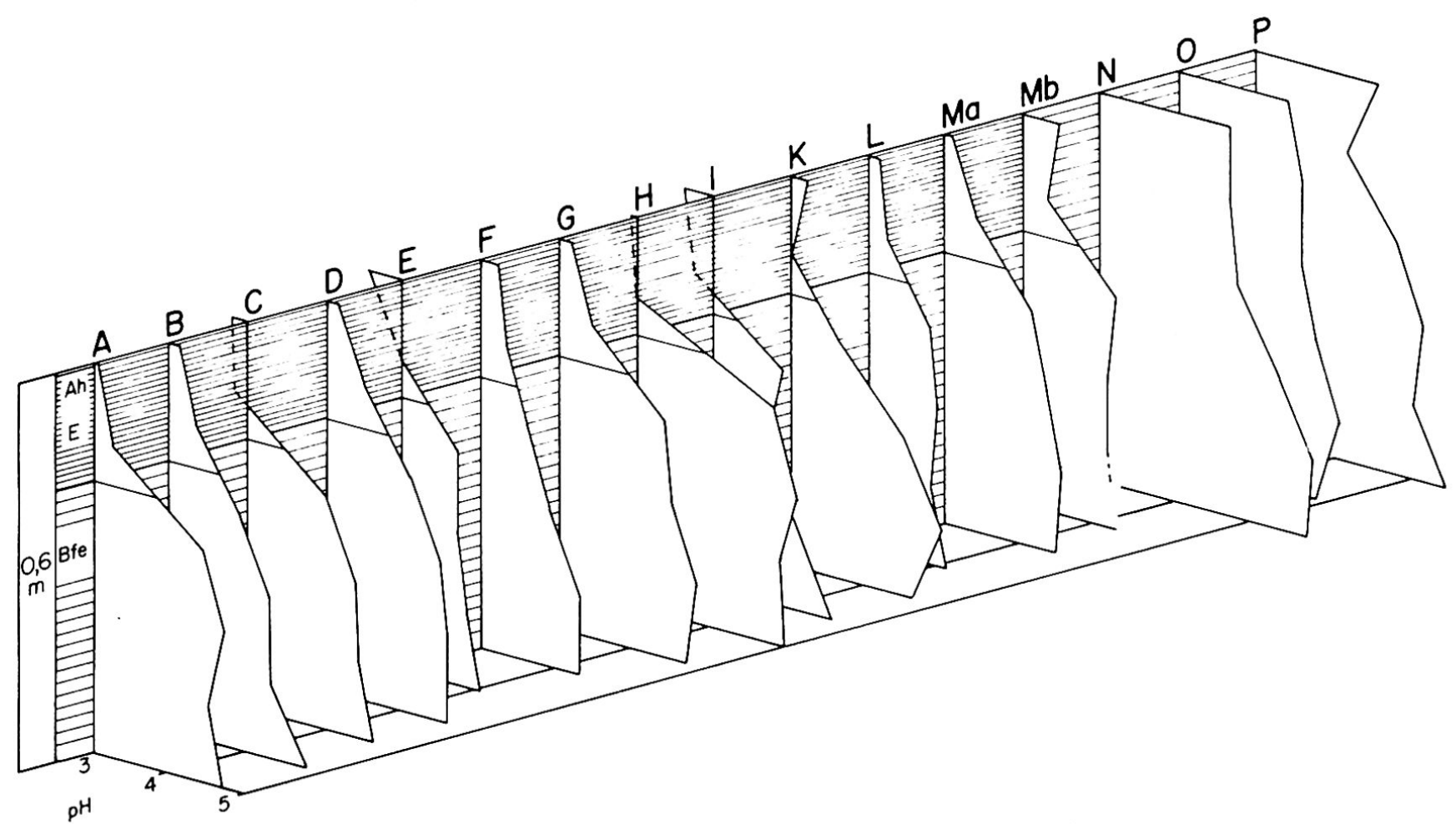

Fig. $4 \mathrm{pH}$-Verhältnisse der Podsole $\mathrm{A}$ bis $\mathrm{M}$ und der Ranker $\mathrm{N}$ bis $\mathrm{P}$. 
kleinsten Teil ausmacht, zu verzerrten Resultaten und Profilverhältnissen führen kann. Es ist also auf diese Weise nicht möglich, irgendwelche Bilanzierungen durchzuführen oder auch nur absolute Gehalte miteinander zu vergleichen.

Aus dem bereits schwach sauren Ausgangsmaterial werden im Lauf der Zeit immer mehr basisch wirkende Kationen durch das Sickerwasser fortgeführt. Die mit der Zeit vermehrt an den Bodentauschern auftretenden Protonen und (sauer wirkenden) Aluminiumionen müßten zu einer Abnahme der Basensättigung und zu einem Absinken des $p H$-Wertes führen. Untersuchungen zur Basensättigung führten zu keinen vernünftigen Resultaten, aber auch die Bestimmung der pH-Werte (Fig. 4) belegt nur, daß alle Podsole offenbar sehr tiefgründig versauert sind. Bei den Podsolprofilen lassen sich keine eindeutigen Unterschiede feststellen, sogar das Profil Mb hebt sich kaum von den übrigen ab. Einzig die drei Ranker $(\mathrm{N}, \mathrm{O}, \mathrm{P})$ unterscheiden sich durch ihren geringen Versauerungsgrad von den Podsolen.

Bei der Verteilung der organischen Substanz interessierte in erster Linie der Minimum-MaximumEffekt (vgl. Fig. 3), wie er durch Verlagerungsvorgänge hervorgerufen wird. FRANZMEIER et al. (1963) konnten beispielsweise feststellen, da $\beta$ eine solche Humusverlagerung erst in etwa 8000 Jahre alten Böden nachzuweisen ist, wogegen in $3000 \mathrm{Jahre}$ alten Böden ein solcher Prozeß noch nicht festgestellt werden konnte. Unsere Untersuchungen (Fig. 5) ergaben jedoch ganz andere Resultate, insbesondere wenn man den maximal 1000 Jahre alten Boden Mb betrachtet, der be- reits eine Podsoldynamik zeigt. Diese Feststellung deckt sich sowohl mit der Ansicht KINGS, wonach beim Steingletscher auf frühneuzeitlichen Moränen eine schwache Podsolierung zu sehen war, als auch mit der Meinung von ZECH und wILKE (1977), die die Böden im Zillertal auf ebenfalls frühneuzeitlichen Moränen als «podsolige alpine Braunerden» ansprechen.

Wie somit die Verhältnisse beim $\mathrm{pH}$-Wert und beim Humus zeigen, müssen die erwarteten Differenzierungen in den ersten hundert Jahren der Bodenentwicklung auftreten, nachher haben diese Böden für diese Kriterien bereits ein «Reifestadium» erreicht. Hervorzuheben ist aber, da $\beta$ dies nur für Böden auf kristallinem Ausgangsmaterial gilt und nicht etwa für karbonathaltige Böden, bei denen der pH-Wert beispielsweise lange Zeit im Neutralbereich verharren kann.

Die weiteren Untersuchungen konzentrieren sich deshalb auf die Sesquioxide Eisen (Fe) und Aluminium(Al). Wenn man von der Überlegung ausgeht, da $B$ mit zunehmender Verwitterungsdauer immer mehr $\mathrm{Fe}$ aus den primären Mineralien freigesetzt und in Form von metallorganischen Komplexen vom E- in den BfeHorizont verlagert wird, so müßte der Anteil dieses pedogenen $\mathrm{Fe}\left(\mathrm{Fe}_{\mathrm{d}}\right)$ am Gesamtgehalt $\left(\mathrm{Fe}_{\mathrm{t}}\right)$ allmählich zunehmen, wie dies beispielsweise TORRENT et al. (1980) an einer Flußterrassensequenz in Spanien sehr schön zeigen konnten. Zu ähnlichen Aussagen gelangen auch BURGER und FRANZ (1969) an der Pasterze. Leider ergab sich im Morteratschgebiet aber keine Korrelation zwischen dem Verhältnis $\mathrm{Fe}_{\mathrm{d}} / \mathrm{Fe}_{\mathrm{l}}$ und der Bodensequenz. Dieses negative Resultat wird aber

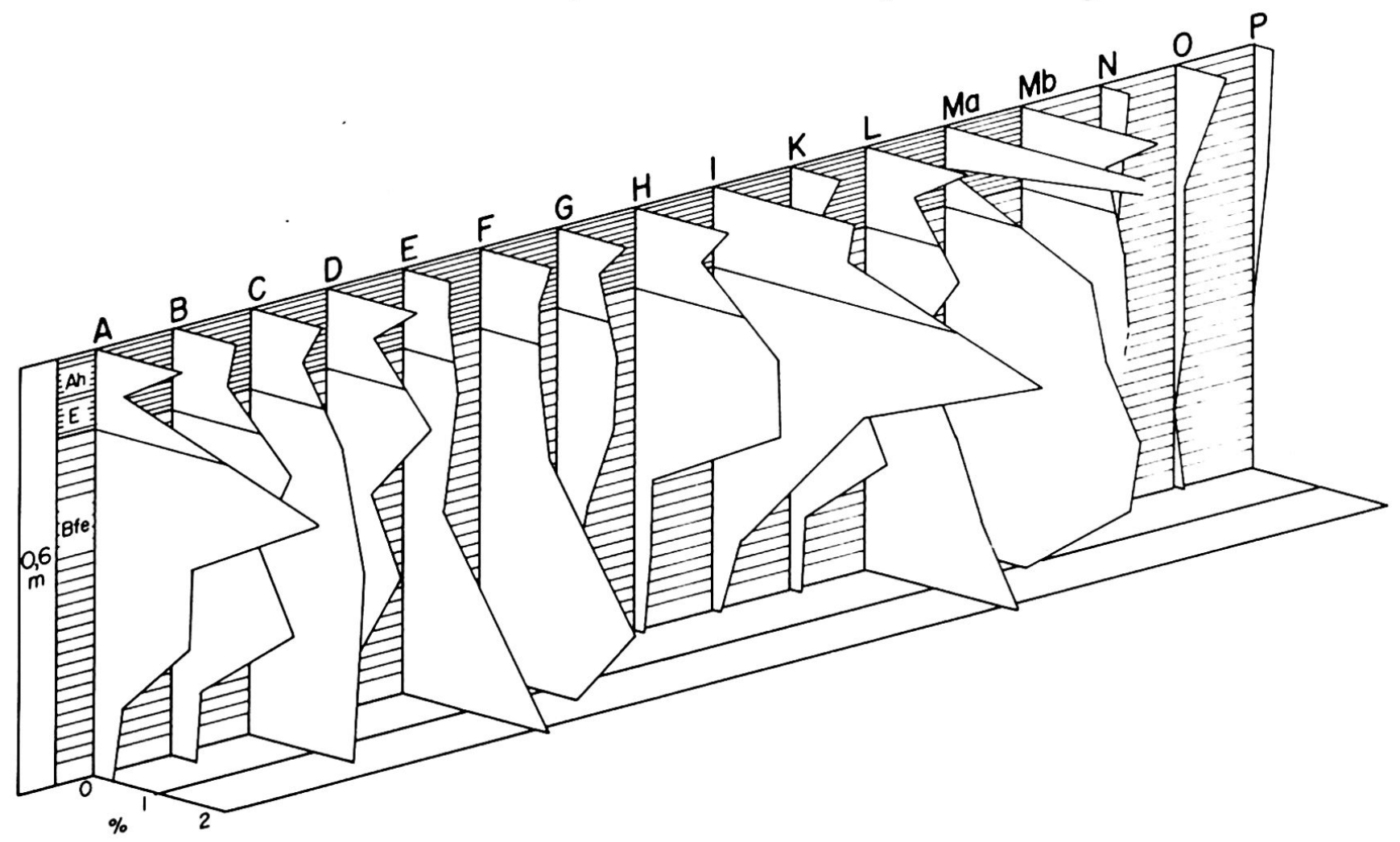

Fig. 5 Gehalt der Profile A bis P an org. Kohlenstoff (in Gewichtsprozenten der Feinerde). Aus zeichnerischen Gründen wurden alle Werte von Ma um 1,4\% vermindert! 


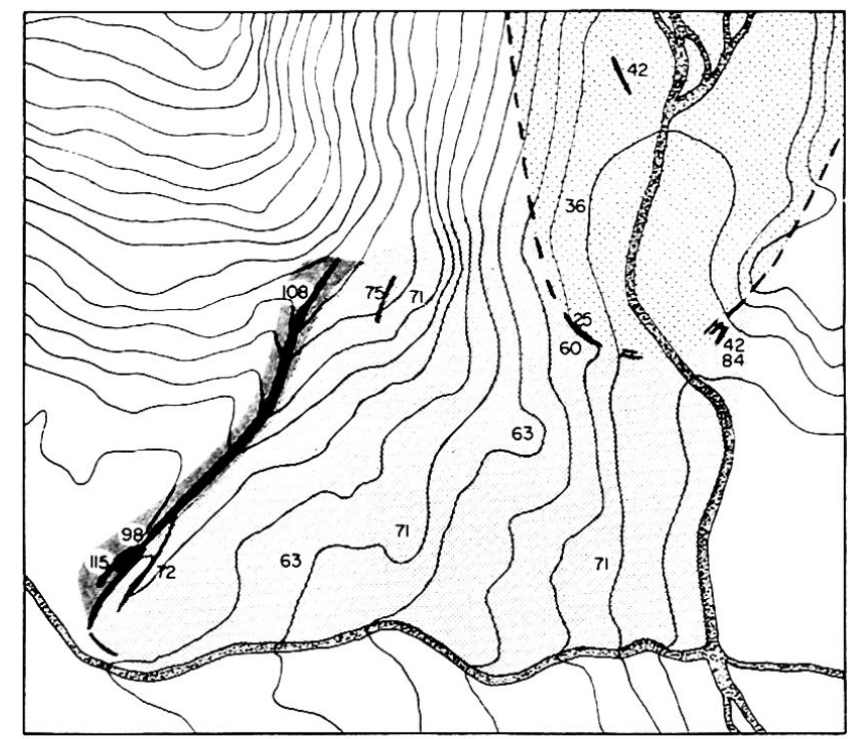

Fig. 6 Die $\mathrm{Al}_{d} / \mathrm{Fe}_{d}$-Verhältnisse (zur Vereinfachung mit 100 multipliziert) ergeben im Morteratschgebiet eine Dreigliederung in die äußersten Stände mit Werten nahe bei 100 oder darüber, in das eigentliche Gletschervorfeld inkl. Mb (Werte unter 50) und einen dazwischen liegenden Bereich mit Werten zwischen 50 und 90.

wahrscheinlich durch den fehlenden Volumenbezug verursacht.

Eine in der Literatur oft zitierte Methode, die Bestimmung des sog. "Aktivitätsgrades» des pedogenen $\mathrm{Fe}$, wurde nach anfänglich positiven Ergebnissen weitergetestet und führte schließlich zu einem Mißerfolg, wobei hier die Ursache bei der organischen Substanz liegen dürfte, die möglicherweise einen kristallisationshemmenden Einfluß ausübt (vgl. BLUME und SCHWERT. MANN, 1969).

Das ebenfalls in die Untersuchungen miteinbezogene Al zeigt grundsätzlich kein anderes Verhalten als das $\mathrm{Fe}$, doch ist zu vermerken, daß das $\mathrm{Al}_{\mathrm{d}}$ nicht dem gesamten pedogenen Anteil entspricht 1 ). Auffällig bei Fig. 3 ist nun aber das hohe $\mathrm{Fe}_{\mathrm{d}} / \mathrm{Fe}_{\mathrm{t}}$-Verhältnis verglichen mit dem $\mathrm{Al}_{\mathrm{d}} / \mathrm{Al}_{\mathrm{t}}$-Verhältnis, und man kann sich eigentlich unschwer ausrechnen, daß bei sehr lang andauernder Verwitterung der Anteil des freiwerdenden Aluminiums $\left(\mathrm{Al}_{d}\right)$ verstärkt zunehmen muß. Es zeigt sich auch bei der Untersuchung der Einzelprofile eindeutig, daß die äußersten drei Böden einen im Vergleich zum Fe- recht hohen Al-Gehalt besitzen (Verhältnis $\mathrm{Al}_{d} / \mathrm{Fe}_{\mathrm{d}}$ um 1 oder größer), wogegen die jüngsten Profile $\mathrm{Mb}, \mathrm{N}, \mathrm{O}, \mathrm{P}$ mit sehr niedrigen Verhältnissen auffallen (kleiner als 0,5). Diese Profilmittelwerte (berechnet aus: mittlerer $\mathrm{Al}_{\mathrm{d}}$-Gehalt des Bfe-Horizontes/mittlerer $\mathrm{Fe}_{\mathrm{d}}$-Gehalt des Bfe-Horizontes) sind in Fig. 6 dargestellt. Am deutlichsten zeigt sich der Unterschied bei der Lokalität $\mathrm{M}$, wo das ältere Profil Ma ein mittleres Verhältnis von 0,84 und das junge Profil $\mathrm{Mb}$ ein solches von 0,42 aufweist.

Eine erste Vermutung, wonach dieses Verhältnis auf das ursprüngliche Verteilungsmuster im Ausgangs- material zurückzuführen wäre, konnte nicht bestätigt werden. Es besteht somit nun die rein empirisch begründete Aussage, daß der pedogene Al-Anteil mit zunehmendem Alter relativ zum pedogenen $\mathrm{Fe}$-Anteil zunimmt. Auf mögliche Ursachen soll weiter unten eingegangen werden.

Dieses Ergebnis würde bedeuten, daß im Morteratschgebiet drei verschieden alte Moränenserien bestehen, zu deren zeitlichen Einstufung sich grundsätzlich zwei Hypothesen aufstellen lassen:

1. Die äußeren Moränen sind älter als egesenzeitlich (von BEELER, 1977, konnten beispielsweise daunzeitliche Moränen des Morteratschgletschers nirgends mit einiger Sicherheit nachgewiesen werden). Die Egesenstände hätten dann allerdings an dieser Stelle praktisch die gleiche maximale Ausdehnung besessen.

2. Die äußeren Wälle sind in die Egesenzeit zu stellen (Languard-Stand von BEELER). Dann müßte der Morteratschgletscher aber im Postglazial noch einmal praktisch die gleiche Ausdehnung erreicht haben.

Vor allem die zweite Hypothese paßt nicht ganz zu den heutigen Kenntnissen, nach denen sich die meisten Gletscher offensichtlich schon im frühen Postglazial (Präboreal) auf die neuzeitliche Größenordnung zurückgezogen haben sollen.

\section{Vergleichsuntersuchungen}

Eine Überprüfung unserer Resultate wurde in drei Gebieten vorgenommen (Fig. 7). Im hinteren Dischmatal, dem Arbeitsgebiet von A. VOEGELI (1976 und Diss. in Bearbeitung), wurden 19 Profile auf spätglazialen Moränen untersucht, wobei von ähnlichen Bodenbildungsbedingungen wie im Morteratschgebiet aus-

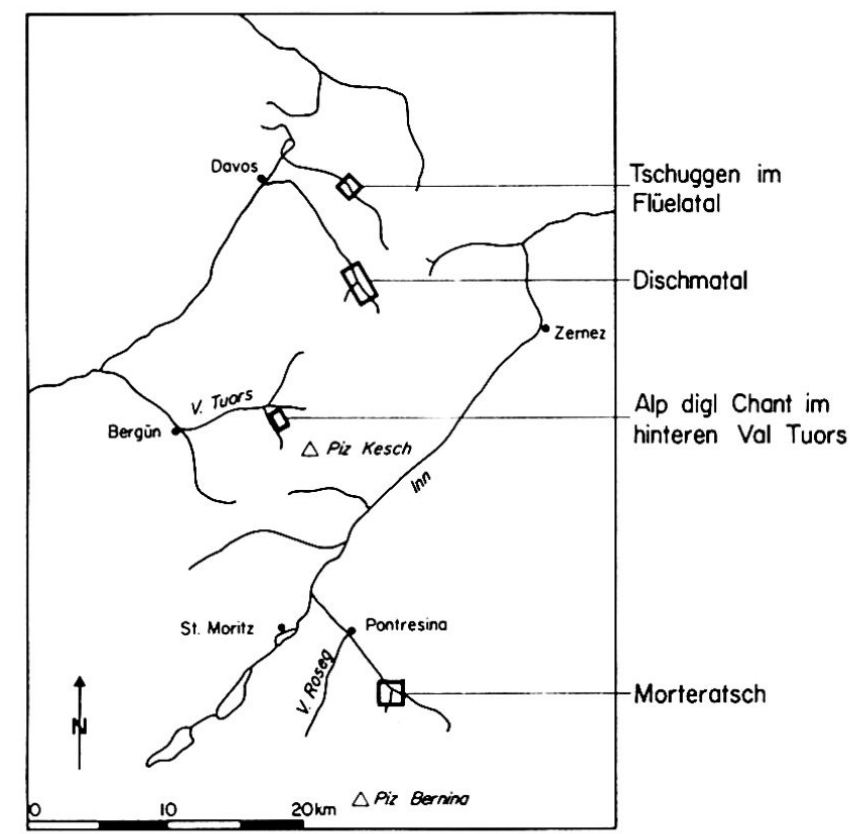

Fig. 7 Übersichtsskizze zur Lage der Untersuchungsgebiete. 
gegangen werden kann. Ohne auf Details einzugehen, kann festgehalten werden, daß die Mittelwerte dieser 19 Profile (Bfe-Horizont) zwischen 0,49 und 1,06 schwanken, die Mittelwerte der Moränen (gewonnen aus 1-5 Einzelprofilen) zwischen 0,62 und 0,82. Diese Zahlen belegen somit die gleiche Größenordnung wie im Morteratschgebiet, wobei sich allerdings keine Sequenz von den jüngsten zu den ältesten Moränen ergibt. Die Untersuchungen von voEgeli belegen aber für das Postglazial an verschiedenen Stellen Phasen stark erhöhter morphologischer Aktivität (nachträglich wieder überschüttete Holzkohlenreste, solifluidale Überlagerung von ehemaligen Böden), so $\mathrm{da} ß$ mit einiger Wahrscheinlichkeit in diesem Gebiet die heute sichtbaren Bodenbildungen nicht dem Alter der morphologischen Formen entsprechen.

Im hinteren Val Tuors, dem Arbeitsgebiet von M. MAISCH (in Bearbeitung), finden sich bei der Alp digl Chant zwei Serien von Moränenständen: Eine innere Serie von deutlich ausgeprägten Wällen, die den Egesenständen entsprechen dürften, und eine etwas weiter außen liegende Serie von undeutlich ausgebildeten, überprägten Wällen aus der Daunzeit. Auf jeder Serie wurden zwei Podsolprofile untersucht, wobei sich zeigt, daß sich das $\mathrm{Al}_{d} / \mathrm{Fe}_{d}-$ Verhältnis mit Mittelwerten bei den jüngeren Moränen von 0,68 und 0,55 nicht von denen der älteren Moränen $(0,61$ und 0,65$)$ abhebt. Zwar ist damit wieder die gleiche Größenordnung wie im Morteratschgebiet und im Dischmatal belegt, doch kommen die eigentlich erwarteten höheren Werte der daunzeitlichen Profile absolut nicht zum Ausdruck. Dies wiederum ist aber verständlich, sind doch die Daunmoränen gerade durch ihren verwaschenen und überprägten Eindruck charakterisiert, was darauf zurückgeführt werden kann, daß diese Wälle bei den späteren Egesenvorstößen erosiv oder solifluidal überprägt und damit auch allfällige Bodenbildungen auf diesen Wällen wieder in Mitleidenschaft gezogen oder sogar zerstört wurden. Die Frage, warum möglicherweise im Morteratschgebiet dieses Phänomen nicht zu beobachten ist, kann höchstens mit der extremen Grobblockigkeit des dort abgelagerten Materials erklärt werden.

Drei weitere Profile auf einer wahrscheinlich im Spätglazial abgelagerten Moräne bei Tschuggen im Flüelatal (VUAGNEUX, 1976) ergaben mittlere $\mathrm{Al}_{\mathrm{d}} / \mathrm{Fe}_{\mathrm{d}}$-Verhältnisse von $0,37,0,50$ und 0,51 , also sehr niedrige Werte für die deutlich ausgebildeten Podsole. An dieser Lokalität sind starke Störungen der Bodenbildungen allerdings offensichtlich (vermutete Waldbrandphase im älteren Subatlantikum, solifluidale Verlagerungen und teilweise Beeinflussung der Profile durch Grundwasser).

\section{Interpretation der Ergebnisse}

In den Untersuchungsgebieten Morteratsch, Dischmatal und Val Tuors ergibt das mittlere $\mathrm{Al}_{d} / \mathrm{Fe}_{\mathrm{d}}$-Verhälnis der Bfe-Horizonte von Podsolen insofern ein ver- nünftiges Alterskriterium, als mit doch vielfältigen morphologischen Prozessen im ausgehenden Spätund im Postglazial gerechnet werden muß. Es ist aber immer noch unklar, ob es sich bei diesem Verhältnis um eine zufällige «Korrelation» mit dem Alter handelt, oder ob eine sinnvolle Erklärung für dieses Resultat gefunden werden kann. Wie schon erwähnt, hat das $\mathrm{Al}_{\mathrm{d}} / \mathrm{Fe}_{\mathrm{d}}$-Verhältnis direkt nichts mit dem im Gestein nachgewiesenen Verhältnis $\left(\mathrm{Al}_{\mathrm{t}} / \mathrm{Fe}_{\mathrm{t}}\right)$ zu tun, wobei nun aber ein indirekter Einfluß der Gesteinszusammensetzung auf die Freisetzung von $\mathrm{Fe}$ und $\mathrm{Al}$ in Betracht gezogen werden muß, wie das folgende, theoretische Beispiel zeigt:

Ausgangspunkt der Überlegung ist ein Korngemisch von feingemahlenem Muskovit und Biotit, das der Verwitterung ausgesetzt wird. Nach SCHEFFER und SCHACHTSCHABEL (1970) betragen die mittleren totalen Gehalte an Fe- und Al-oxiden (Gewichts-\%):

$\begin{array}{lll} & \mathrm{Al}_{2} \mathrm{O}_{3} & \mathrm{Fe}_{2} \mathrm{O}_{3}+\mathrm{FeO} \\ \text { Muskovit } & 33,6 & 13,0 \\ \text { Biotit } & 17,1 & 19,0\end{array}$

Aus der Stabilitätsreihe von Mineralien ergibt sich, daß Muskovit im allgemeinen wesentlich stabiler ist als Biotit. Aus einem Korngemisch, das sich somit chemisch durch einen gegenüber dem Eisen sehr hohen Al-Gehalt auszeichnet, muß zu Beginn der Verwitterung mehr Biotit als Muskovit zerfallen, was sich in einer verstärkten Fe-Freisetzung äußert. Erst nach längerer Zeit, wenn auch der Muskovit verstärkt angegriffen wird, sollte ein höherer Anteil des Al bei den pedogenen Oxiden nachzuweisen sein.

Theoretisch wäre es also möglich, aus der Mineralzusammensetzung eines Gesteins und der Stabilitätsreihe der Mineralien die Verwitterung nachzuvollziehen. Es gibt aber einige Gründe, die dieses Unterfangen als fast aussichtslos erscheinen lassen:

- Die mittlere Mineralzusammensetzung ist nur ein Anhaltswert, der von Aufschluß zu Aufschluß oder sogar innerhalb eines Profils beträchtlich variieren kann.

- Bei den extrem unterschiedlichen Korngrößenverhältnissen in Moränen ist die Stabilitätsreihe von geringem Nutzen.

- Die Stabilitätsreihe selbst ist sehr umstritten. Je nach Autor lautet die Reihe etwas anders. Zudem ist eine solche Reihe klimaabhängig und daher nicht generell anwendbar.

Unter der Voraussetzung, daß $\mathrm{Fe}$ und $\mathrm{Al}$ nicht selektiv aus dem Bodensystem mit dem Sickerwasser entfernt werden (was kaum anzunehmen ist), kann nun aber auf andere Weise ein Indiz dafür gefunden werden, daß möglicherweise in einer initialen Phase der Bodenbildung das $\mathrm{Fe}$ und erst später das $\mathrm{Al}$ überwiegt. Es ist ja bekanntlich so, daß die im Unterboden immobilisierten Sesquioxide sich in Form von Krusten auf den Steinen niederschlagen. Wenn nun eine solche Kruste schichtweise analysiert werden könnte, so müßte sich eine Verschiebung zugunsten des $\mathrm{Al}$ im 


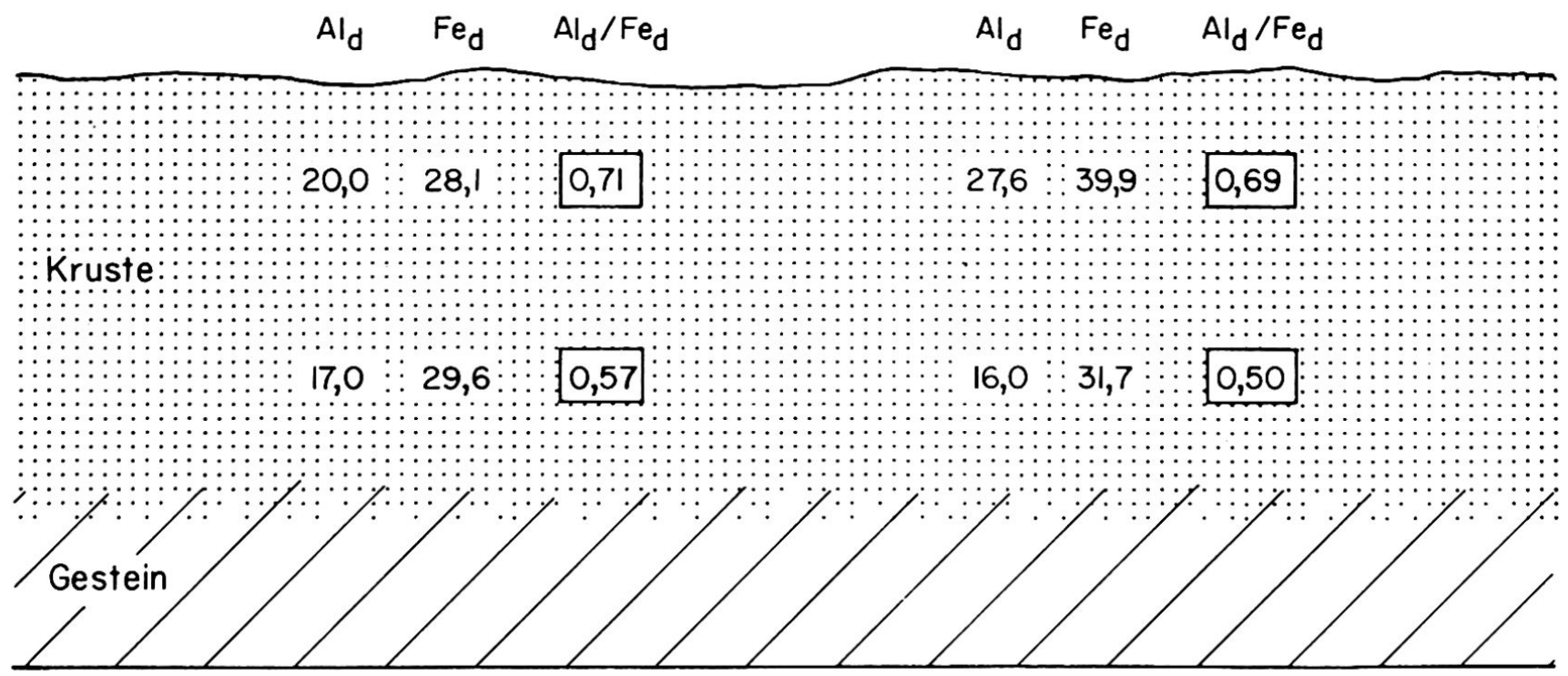

Fig. 8 Schematische Darstellung des im Text beschriebenen Versuches, das $A l_{d} / \mathrm{Fe}_{d}$-Verhältnis im inneren und äußeren Bereich der Gesteinskruste (Bfe-Horizont) zu ermitteln. Es handelt sich um zwei Parallelversuche (a und b) aus derselben Moräne bei der Alp digl Chant. Angaben von $\mathrm{Al}_{d}$ und $\mathrm{Fe}_{d}$ in Gewichts-\%o.

Verlauf der Zeit nachweisen lassen. Zu diesem Zweck wurden von etwa einem Dutzend Steinen aus dem Bfe-Horizont eines Podsolprofils aus dem Val Tuors mit einem scharfen Messer zuerst nur die äußersten Partien der feinen, rotbraunen Kruste und für eine zweite Analyse bei den gleichen Steinen der Rest der Kruste bis auf die graue Gesteinsoberfläche abgekratzt. Die in diesen beiden Proben ermittelten $\mathrm{Al}_{d^{-}}$ und $\mathrm{Fe}_{\mathrm{d}}-\mathrm{Gehalte}$ sowie die entsprechenden $\mathrm{Al}_{\mathrm{d}} / \mathrm{Fe}_{\mathrm{d}^{-}}$ Verhältnisse sind in Fig. 8a dargestellt. Um eine reine Zufälligkeit auszuschalten, wurde mit einer zweiten Serie von Steinen (aus dem gleichen Horizont) der Versuch wiederholt (Fig. 8b).

Als Resultat aus diesen recht groben Versuchen läßt sich festhalten, da $B$ in beiden Fällen das $\mathrm{Al}_{d} / \mathrm{Fe}_{\mathrm{d}}$-Verhältnis im innern Bereich der Kruste (initiale Bodenbildung) eindeutig niedriger ist als im äußeren Teil (weiter fortgeschrittene Bodenbildung). Natürlich ist es auf diese Weise nicht möglich, eine angelagerte Kruste von einer in situ-Bildung bei der Gesteinsverwitterung zu unterscheiden. Interessant sind die Werte in Fig. $8 \mathrm{a}$ aber auch darum, weil der $\mathrm{Fe}_{\mathrm{d}}$-Gehalt im inneren Krustenbereich höher ist $(29,6 \%)$ als im äußeren Teil $(28,1 \%$ ) . Unter der Annahme einer gleichmäßigen $\mathrm{Fe}$-Verteilung über die ganze Dicke der Kruste müßte bei der Analyse ein niedrigerer Wert im inneren Teil erwartet werden, bedingt durch den in dieser untersuchten Probe doch schon beträchtlichen Anteil an unverwittertem Gesteinsstaub, der ja kein $\mathrm{Fe}_{\mathrm{d}}$ enthalten darf. Die erwähnten Werte lassen nun darauf schließen, daß im Verlauf der Zeit eher mit einer Abnahme des angelagerten Eisens gerechnet werden muß, wogegen über das Verhalten des
Aluminiums nichts ausgesagt werden kann. Es läßt sich mit dieser groben Versuchsanordnung also festhalten, da $B$ das $\mathrm{Al}_{d} / \mathrm{Fe}_{\mathrm{d}}$-Verhältnis mit zunehmendem Alter des Bodens größer wird, und daß diese Änderung primär mit einer Abnahme des Eisens in Verbindung gebracht werden kann. Nach diesem positiven Ergebnis wurde eine noch genauere Untersuchung einer Gesteinskruste aus demselben Horizont durchgeführt. Als Methode wurde die an das Rasterelektronenmikroskop gekoppelte energiedispersive Röntgenanalyse (EDAX) ${ }^{2)}$ gewählt, die ja heute zu den Standardmethoden zählt. Als Resultat aus dieser semiqantitativen Analyse geht hervor, da $B$ im Bereich der äußeren Kruste das $\mathrm{Al}$ gegenüber dem $\mathrm{Fe}$ deutlich dominanter ist als im inneren Bereich. Dies ist somit erneut eine Bestätigung des Ergebnisses unserer Bodenuntersuchungen.

$\mathrm{Da}$ es eigentlich im Verlauf der Zeit zu einer AlDominanz über das Fe kommen muß, ergibt sich auch aus dem mittleren Verhältnis $\mathrm{Al}_{\mathrm{l}} / \mathrm{Fe}_{\mathrm{t}}$ des Gesteins im Morteratschgebiet, das bei 2,6 liegt. Je mehr wir uns dem Stadium einer (fiktiven) totalen Verwitterung nähern, um so mehr müßte sich das $\mathrm{Al}_{d} / \mathrm{Fe}_{\mathrm{d}}$-Verhältnis dem $\mathrm{Al}_{\mathrm{t}} / \mathrm{Fe}_{\mathrm{t}}-$ Verhältnis angleichen. Dies immer unter der Voraussetzung, daß kein selektiveı lirlust im System stattfindet.

Eine der wenigen direkten Bestätigungen unserer Ergebnisse findet sich beispielsweise bei AALTONEN (zit. in JAUHIAINEN, 1972 a, p. 11): "...the relative abundance of aluminium in relation to iron are typical of an old-soil podzol mineral colloid complex...». Dies wirft nun auch die Frage nach der überregionalen Vergleichbarkeit der $\mathrm{Al}_{\mathrm{d}} / \mathrm{Fe}_{\mathrm{d}}$-Verhältnisse auf. 
Aus unseren Untersuchungen läßt sich ableiten, daß altersmäßig vergleichbare Moränen auch in der GröBenordnung vergleichbare $\mathrm{Al}_{d} / \mathrm{Fe}_{d}$-Verhältnisse aufweisen. $\mathrm{Da} B$ die gefundene Größenordnung für die kristallinen Alpen offensichtlich realistisch ist, belegen auch die Untersuchungen von BLASER (1973) an Podsolen im südlichen Tessin: Das mittlere $\mathrm{Al}_{1} / \mathrm{Fe}_{\mathrm{d}^{-}}$ Verhältnis ${ }^{1)}$ aus den $\mathrm{B}_{\mathrm{s}}-$ bzw. $\mathrm{B}_{\mathrm{s}} \mathrm{C}$-Horizonten seiner neun Profile ergibt einen Wert von 0,97. Die zeitliche Stellung dieser Profile ergibt sich aber höchstens aus dem Hinweis, daß diese Böden in Hangschuttmassen gefunden wurden, denen stellenweise Reste von Moränenmaterial beigemengt sind. Ein Vergleich mit einer bodenkundlichen Arbeit aus Finnland (JAUHIAINEN 1972b) macht allerdings deutlich, da $\beta$ dieses Verhältnis zu ganz anderen Werten führen kann und somit eine kritiklose Übertragung auf andere Regionen nicht möglich zu sein scheint.

\section{Zusammenfassung}

Im Morteratschgebiet kann aufgrund unserer Untersuchungen gezeigt werden, daß sich verschieden alte Podsole höchstens durch das $\mathrm{Al}_{d} / \mathrm{Fe}_{\mathrm{d}}$-Verhältnis unterscheiden, wobei mit zunehmendem Alter dieses Verhältnis größer wird. Kein Zusammenhang mit dem Alter ergeben Untersuchungen von Horizontmächtigkeiten, pH-Wert, Verlagerung der organischen Substanz, Aktivitätsgrad und absolute Gehalte an Sesquioxiden. Untersuchungen von Böden im Dischmatal und im Val Tuors ergaben zumindest keinen Widerspruch zum gefundenen Resultat, im Flüelatal sind die Verhältnisse sowohl in bodenkundlicher als auch in morphologischer Hinsicht unklar.

Eine Bestätigung unserer Resultate ergeben die Analysen von Gesteinskrusten aus dem Bfe-Horizont, sowohl auf naßchemische Weise als auch durch die energiedispersive Röntgenanalyse.

Die Ursache für die im Lauf der Zeit zugunsten des Al verschobenen Verhältnisse muß wahrscheinlich bei der Verwitterung gesucht werden, doch führten Überlegungen im Zusammenhang mit der unterschiedlichen Verwitterungsresistenz der das Gestein aufbauenden Minerale zu keinem konkreten Ergebnis.

Eine detaillierte Chronosequenz bei Podsolbildungen konnte nicht ermittelt werden. Dies hängt sicher auch damit zusammen, daß sich die Gletscher wahrscheinlich schon zu Beginn des Postglazials auf neuzeitliche Größenordnung zurückgezogen haben, daß also alle Zeugen der letzten 9000-10000 Jahre in einem sehr kleinen, kompliziert aufgebauten Bereich gesucht werden müssen. Hinzu kommt, daß sich ein deutliches Podsolprofil in einigen hundert bis maximal $1000 \mathrm{Jah}$ ren entwickelt haben dürfte und somit nur sehr genaue Analysen über die weitere Entwicklung der Profile Aufschluß geben können.

\section{Anhang: Analytische Untersuchungsmethoden}

- pH-Wert: Messung mittels Glaselektrode in $\mathrm{n} \mathrm{KCl}$ Lösung (Verhältnis lufttrockene Feinerde: $\mathrm{KCl}$ Lösung $=1: 2,5$ );

- organischer Kohlenstoff: nasse Veraschung mit $\mathrm{K}_{2} \mathrm{Cr}_{2} \mathrm{O}_{7}$ nach Walkley-Black (in JACKSON, 1958) ohne äußere Wärmezufuhr;

- oxalatlösl. Eisen $\left(\mathrm{Fe}_{\mathrm{o}}\right)$ : nach der Methode von Tamm, mod. nach SCHWERTMANN (1959);

- dithionitlösl. Eisen und Aluminium $\left(\mathrm{Fe}_{d}, \mathrm{Al}_{d}\right)$ : Extraktion mit Dithionit-Citrat bei $\mathrm{pH} 7,3$ nach MEHRA Und JACKSON (1960);

- totales Eisen und Aluminium $\left(\mathrm{Fe}_{\mathrm{t}}, \mathrm{Al}_{\mathrm{t}}\right)$ : Aufschluß mit $\mathrm{HF}$ und $\mathrm{HClO}_{4}$.

Alle Mengenbestimmungen erfolgten titrimetrisch.

\section{Anmerkungen}

1) Der Gesamtgehalt eines Bodens an «freiem» $\mathrm{Al}$ $\left(\mathrm{Al}_{l}\right)$ müßte besser im $\mathrm{NaOH}$-Auszug ermittelt werden. Gemäß unseren Erfahrungen liegt der $\mathrm{Al}_{d-}$ Gehalt rund $25 \%$ unter dem $\mathrm{Al}_{1}$-Gehalt, doch ändert dieser Unterschied nichts am Verteilungsmuster (vgl. dazu auch BLume und SCHWERTMANN, 1969), so daß auf einen zusätzlichen Auszug verzichtet wurde.

2) Bei diesem Verfahren wird die emittierte Röntgenstrahlung gemessen, die dadurch entsteht, $\mathrm{da}$ die schnellen Elektronen der Bestrahlungsquelle die Atome des zu untersuchenden Präparats ionisieren können (Entfernung von Elektronen). Beim «Nachrutschen» von Elektronen aus höheren Niveaus wird die Energiedifferenz als Strahlung emittiert. Dieses für jedes Element charakteristische Emissionsspektrum kann für die Analyse verwendet werden.

\section{Zitierte Literatur}

BEELER F. (1977): Geomorphologische Untersuchungen am Spät- und Postglazial im Schweizerischen Nationalpark und im Berninapaßgebiet (Südrätische Alpen). Erg. wiss. Unters. Nat. park, XV, p. 131-276.

BLASER P. (1973): Die Bodenbildung auf Silikatgestein im südlichen Tessin. Schweiz. Anst. f. d. forstl. Versuchswesen, 49 (3), p. 251-340.

BLUME H. P. und SCHWERTMANN U. (1969): Genetic evaluation of profile distribution of Aluminium, Iron, and Manganese Oxides. Soil Sci. Soc. Amer. Proc., 33, p. $438-444$.

BURGER R. (1972): Die Böden der Pasterzenlandschaft im Glocknergebiet. Mitt. d. Österr. Bodenkundl. Ges., 16 , p. 23-92. 
BURGER R. und FRANZ H. (1969): Die Bodenbildung in der Pasterzenlandschaft. Wiss. Alpenvereinshefte, 21, p. 253-264.

FRANZMEIER D. P., WHITESIDE E. P., and MORTLAND M. M. (1963): A chronosequence of podzols in Northern Michigan. Mich. Agr. Expt. Sta. Quart. Bul., 46, p. 1-57.

JACKSON M. L. (1958): Soil chemical analysis. Constable \& Co., London, 498 pp.

JAUHIAINEN E. (1969): On soils in the boreal coniferous region. Fennia, 98 (5), 123 pp.

JAUHIAINEN E.(1972b): Structure, C-14-age and environment of an Iron-Humus Podzol under a peat layer. Ann. Acad. Scient. Fenniae, Ser. A, 112, p. 1-16.

JAUHIAINEN E. (1972a): Rate of Podzolization in a dune in Northern Finland. Comment. Phys.-Math. 112, p. 33-44.

KING L. (1974): Studien zur postglazialen Gletscherund Vegetationsgeschichte des Sustenpaßgebietes. Basler Beitr. z. Geogr., 18, 123 pp.

MAYR F. (1964): Untersuchungen über Ausma $B$ und Folgen der Klima- und Gletscherschwankungen seit dem Beginn der postglazialen Wärmezeit. Zeitschr. f. Geomorph., N. F. 8 (3), p. 257-285.

MEHRA O. P. and JACKSON M. L. (1960): Iron oxide removal from soils and clays by a dithionite-citrate system buffered with sodium bicarbonate. Clays and Clay Minerals, 7, p. 317-327.

PATZELT G. (1973): Die neuzeitlichen Gletscherschwankungen in der Venedigergruppe. Zeitschr. f. Gletscherkde. u. Glazialgeologie, IX (1-2), p. 5-57.

SCHEFFER F. und SCHACHTSCHABEL P. (1970): Lehrbuch der Bodenkunde, Ferd. Enke, Stuttgart, 448 pp. SCHWERTMANN U. (1959): Die fraktionierte Extraktion der freien Eisenoxide in Böden, ihre mineralogischen Formen und ihre Entstehungsweise. Zeitschr. für Pflanzenernähr., Düng., Bodenkunde, 84, p. 194-204.

TORRENT J., SCHWERTMANN U., SCHULZE D. G. (1980): Iron oxide mineralogy of some soils of two river terrace sequences in Spain. Geoderma, 23, p. 191-208.

VOEGELI A. (1976): Untersuchungen postglazialer Gletscherstände im Dischmatal (Davos, Graubünden). Dipl.-arbeit, Man. Geogr. Inst. Univ. Zürich, 35 pp.

VUAGNEUX R. (1976): Untersuchungen spät- und postglazialer Gletscherstände im Raume Flüelapaß. Dipl.Arbeit, Man. Geogr. Inst. Univ.Zürich, 57 pp.

ZECH W. und WILKE B.-M. (1977): Vorläufige Ergebnisse einer Bodenchronosequenzstudie im Zillertal. Mitt. Dtsch. Bodenkundl. Ges., 25, p. 571-586.

\section{Literaturbesprechung}

STINGELIN, A., Oeffentliche Werke im ländlichen Raum - Die Bedeutung der Landumlegung, Schulthess Verlag, 200 S., broschiert, Fr. 34.--

Ausgehend von einem umfassenden, gesamträumlichen Planungsverständnis, wie es seitens der Geographie immer wieder gefordert wurde, greift der Kulturingenieur Stingelin in seiner erweiterten Dissertation ein, vielleicht das heisse Eisen im Planungsrecht an: Das öffentliche Interesse nach Art. 23 BV. Es ist Voraussetzung für allfällige Enteignungsverfahren im Zusammenhang mit der Errichtung öffentlicher Werke. Dieser Bundesverfassungsartikel fristete seit seines Bestehens ein Schattendasein, sei es in der parlamentarischen Diskussion, sei es in der rechtswissenschaftlichen Analyse. Aufgrund der ständigen Zunahme öffentlicher Aufgaben, drängt sich, wie der Autor nachweist, eine neue Definition des öffentlichen Interesses und des Werkes auf. Die neue Definition von Stingelin ist nicht nur überzeugend, sie erscheint geradezu eine Grundvoraussetzung dafür, die in der geographischen Diskussion erfreulicherweise zahlreicher werdenden Wirkungsanalysen von Infrastrukturanlagen zu Grundlagen einer Unweltsverträglichkeitsprüfung $\mathrm{zu}$ machen, denn das öffentliche Werk umfasst nicht nur den körperli- chen Gegenstand, sondern ebenso Betrieb, Unterhalt und Umweltbeeinflussung.

Zwingend ist auch seine Beweisführung, die Nutzungsplanung sei als öffentliches Gut zu behandeln wie ein öffentliches Werk, dem sie durch ihre Anforderungen an Standort, Flächenbedarf und ihre Auswirkungen auf das Grundeigentum durchaus vergleichbar ist. Stingelin bleibt jedoch nicht beim Problemaufriss stehen, er zeigt auch konkrete Wege zur Realisierung. Einlässlich behandelt er das Institut der Landumlegung, stellt dessen herausragende Bedeutung als Instrument des Interessenausgleichs bei der Errichtung öffentlicher Werke dar und gibt Hinweise auf dessen funktionsgerechte Ausgestaltung und Organisation.

Das Buch sollte Pflichtlektüre (und wird als solche bald zum wertvollen Ratgeber) für jeden Planer, Ingenieur, Juristen ob in Verwaltung oder Privatwirtschaft sein. Darüberhinaus eröffnet es dem in Praxis wie in Wissenschaft tätigen Geographen dank seines weitgefassten Rahmens von kulturtechnischen Belangen bis zu bodenrechtlichen Abhandlungen und ökonomischen Fragestellungen interessante Perspektiven für die eigene, geographische Arbeit.
GH $3 / 80$
Gabriela Winkler, Zürich 